\title{
A Generalized Sampling Theorem for Frequency Localized Signals
}

\author{
Edwin Hammerich \\ Ministry of Defence \\ Kulmbacher Str. 58-60, D-95030 Hof, Germany \\ e-mail: edwin.hammerich@ieee.org
}

Dedicated to Professor Abdul J. Jerri on the occasion of his 75th birthday

\begin{abstract}
A generalized sampling theorem for frequency localized signals is presented. The generalization in the proposed model of sampling is twofold: (1) It applies to various prefilters effecting a "soft" bandlimitation, (2) an approximate reconstruction from sample values rather than a perfect one is obtained (though the former might be "practically perfect" in many cases). For an arbitrary finite-energy signal the frequency localization is performed by a prefilter realizing a crosscorrelation with a function of prescribed properties. The range of the prefilter, the so-called localization space, is described in some detail. Regular sampling is applied and a reconstruction formula is given. For the reconstruction error a general error estimate is derived and connections between a critical sampling interval and notions of "soft bandwidth" for the prefilter are indicated. Examples based on the sinc-function, Gaussian functions and B-splines are discussed.
\end{abstract}

Key words and phrases: frequency localization, reproducing kernel Hilbert space, interpolating function, error estimate, generalized Chebyshev inequality, critical sampling interval, generalized sampling theorem

\section{Introduction}

In time-frequency analysis the concept of time-frequency localization is wellknown [15], 8], [12]. It means the approximate concentration of a signal in both time and frequency allowing for tails of strong decay in either dimension. In many areas of communications the concept of (strict) bandlimitation is still prevailing. One reason might be the classical sampling theorem of Whittaker, Kotel'nikov and Shannon [24], [18, [5], 28], 29] where bandlimited signals are 
the basic assumption. This leads to difficulties in practical applications [28] of the classical sampling theorem and researchers have been trying to overcome the entailed complications [3], 66, [9, [19, [25], 26], 28]. The goal of the present work is to contribute to these attempts by loosening the assumption of strict bandlimitation while retaining the model of sampling of the classical sampling theorem: An arbitrary finite-energy input signal is preprocessed by a prefilter, the filter output signal lying in a well-structured space similar to the space of bandlimited signals is ideally sampled at equidistant points of time, finally the complete filter output signal is to be reconstructed. A whole variety of prefilters is described some of which are presumably suitable for practical applications. The price to be paid will be, in general, imperfect reconstruction. Some effort is made to estimate the incurred reconstruction error and to find criteria for the size of the sampling interval to guarantee good reconstruction. This is well in the sense of A. J. Jerri's work on error analysis in sampling theory and applications [18], [19. The chosen approach incorporates from the start some measure of generalized bandwidth for the used prefilter so that eventually the link between that generalized bandwidth (the so-called "soft bandwidth") and a critical sampling interval shall be found. That critical sampling interval would then correspond to the Nyquist interval in case of the classical sampling theorem [24], 29].

The paper is organized as follows. In Section 2 the frequency localization operator (prefilter) is defined and basic assumptions are compiled. In Section 3 the so-called localization space is described. In Section 4 a perfect reconstruction formula for elements of some subspace of localization space is derived. In Section 5 a general error estimate for the reconstruction error is given. As a conclusion, in Section 6 the sampling theorem is presented. In subsections at the end of Section 5 and 6 examples are discussed.

The following notation is used: $L^{2}(\mathbb{R})$ is the space of square integrable functions (or finite-energy signals) $f: \mathbb{R} \rightarrow \mathbb{C}$ with inner product $\left\langle f_{1}, f_{2}\right\rangle=$ $\int_{-\infty}^{\infty} f_{1}(x) \overline{f_{2}(x)} d x$. For the Fourier transform we adopt the convention $\hat{f}(\xi)=$ $(2 \pi)^{-1 / 2} \int_{-\infty}^{\infty} e^{-i x \xi} f(x) d x$, where $x$ denotes time and $\xi$ (angular) frequency. $\mathbb{R}_{+}$denotes the set of positive real numbers.

\section{Frequency Localization Operator}

For an arbitrary finite-energy signal $f \in L^{2}(\mathbb{R})$ frequency localization is performed by an operator $\boldsymbol{P}_{\varphi}: L^{2}(\mathbb{R}) \rightarrow L^{2}(\mathbb{R})$ given by

$$
\left(\boldsymbol{P}_{\varphi} f\right)(x)=\int_{-\infty}^{\infty} f(y) \overline{\varphi(y-x)} d y=\int_{-\infty}^{\infty} e^{i x \xi} \overline{\hat{\varphi}(\xi)} \hat{f}(\xi) d \xi .
$$

We shall call $\boldsymbol{P}_{\varphi}$ prefilter because in practice it would be an anti-aliasing prefilter or a related nonideal acquisition device [26]. Since $\left(\boldsymbol{P}_{\varphi} f\right)(x)=$ 
$\langle f, \varphi(\cdot-x)\rangle, x \in \mathbb{R}$, the prefilter realizes a crosscorrelation of the input signal $f$ with $\varphi$ (rather than a convolution with $\overline{\varphi(-x)}$ ). We make the following assumptions on the prefilter function $\varphi$ :

(i) $\varphi \in L^{2}(\mathbb{R})$

(ii) It holds the generalized moment condition

$$
M_{w}(\varphi)=\int_{-\infty}^{\infty} w(|\xi|)|\hat{\varphi}(\xi)|^{2} d \xi<\infty
$$

where $w=w(\xi)$ for $\xi>0$ is a positive and monotonically increasing weight function of sufficient growth,

$$
w(\xi) \geq c \xi^{1+\epsilon} \quad \forall \xi \in[1, \infty)(c>0, \epsilon>0) .
$$

(iii) For any $\lambda \in \boldsymbol{\Lambda}$, where $\boldsymbol{\Lambda}$ is some non-empty subset of $\mathbb{R}_{+}$, the family of functions $\{\varphi(\cdot-n \lambda) ; n \in \mathbb{Z}\}$ forms a Riesz basis in $L^{2}(\mathbb{R})$.

We refer to [7], 28] concerning Riesz bases and their relevance to signal processing. The Riesz basis condition is equivalent to the existence of positive real numbers $A, B$ (possibly depending on $\lambda$ ) so that

$$
0<A \leq \Lambda \sum_{n=-\infty}^{\infty}|\hat{\varphi}(\xi+n \Lambda)|^{2} \leq B<\infty \text { a.e. }
$$

where $\Lambda=2 \pi / \lambda$. See [2] for a proof in case $\lambda=1$, the argument carries over to arbitrary $\lambda>0$ without changes. Actually, we shall use the Riesz basis condition mostly in form of Ineq. (4).

Because of the upper bound in Ineq. (4) the Fourier transform $\hat{\varphi}$ is bounded almost everywhere on $\mathbb{R}$ so that the range of $\boldsymbol{P}_{\varphi}$ is a subset of $L^{2}(\mathbb{R})$ as presumed. We note that because of condition (3) the prefilter function $\varphi$ is in a Sobolev space [21] $H^{r}(\mathbb{R})=\left\{u \in L^{2}(\mathbb{R}) ; \int|\hat{u}(\xi)|^{2}\left(1+|\xi|^{2}\right)^{r} d \xi<\infty\right\}$ of order $r>\frac{1}{2}$. Thus, by reason of the Sobolev embedding theorem, $\varphi$ will necessarily be a continuous function.

In our paper monomial weights $w(\xi)=|\xi|^{s}, s>1$, and Gaussian weights $w(\xi)=\exp \left(s \xi^{2}\right), s>0$, will be used. As prefilter functions $\varphi$ the sinc-function, Gaussian functions and B-splines will be taken.

\section{Localization Space}

The localization space $\mathcal{P}_{\varphi}$ of the prefilter $\boldsymbol{P}_{\varphi}$ corresponds to the space of bandlimited signals in case of an ideal low-pass filter and is defined as the range of $\boldsymbol{P}_{\varphi}$,

$$
\mathcal{P}_{\varphi}=\left\{g=\boldsymbol{P}_{\varphi} f ; f \in L^{2}(\mathbb{R})\right\} .
$$


$\mathcal{P}_{\varphi}$ is a linear, not necessarily closed subspace of $L^{2}(\mathbb{R})$, it even may be dense in $L^{2}(\mathbb{R})$. Moreover, the localization space is invariant with respect to arbitrary translations $g \mapsto g(\cdot-t), t \in \mathbb{R}$. The function $\Phi \in \mathcal{P}_{\varphi}$ defined by

$$
\Phi=\boldsymbol{P}_{\varphi} \varphi \stackrel{\text { Fourier }}{\longleftrightarrow} \hat{\Phi}(\xi)=\sqrt{2 \pi}|\hat{\varphi}(\xi)|^{2}
$$

will be of importance. Since $\Phi(x)=\langle\varphi, \varphi(\cdot-x)\rangle, x \in \mathbb{R}$, it is the autocorrelation function of $\varphi$. On $\mathcal{P}_{\varphi}$ we define the inner product

$$
\left\langle g_{1}, g_{2}\right\rangle_{\varphi}=\frac{1}{2 \pi} \int_{\operatorname{supp} \hat{\varphi}} \hat{g}_{1}(\xi) \overline{\hat{g}_{2}(\xi)}|\hat{\varphi}(\xi)|^{-2} d \xi
$$

with corresponding norm $\|g\|_{\varphi}=\sqrt{\langle g, g\rangle_{\varphi}}$.

The reproducing kernel Hilbert space (RKHS) property of localization space $\mathcal{P}_{\varphi}$ will be described in the next theorem. The shorthand notation $L^{2}(\mathbb{R} \mid \operatorname{supp} \hat{\varphi})$ for the closed subspace $\left\{f \in L^{2}(\mathbb{R}) ; \operatorname{supp} \hat{f} \subseteq \operatorname{supp} \hat{\varphi}\right\}$ of $L^{2}(\mathbb{R})$ will be used. We remark that $L^{2}(\mathbb{R} \mid \operatorname{supp} \hat{\varphi})=L^{2}(\mathbb{R})$ in case of a Gaussian function or a B-spline $\varphi$ (see Section 6.1).

Proposition 1 The linear space $\mathcal{P}_{\varphi}$ endowed with the inner product (5) is an $R K H S$. The reproducing kernel is $K(x, y)=\Phi(y-x)$. $\boldsymbol{P}_{\varphi}$ maps $L^{2}(\mathbb{R} \mid \operatorname{supp} \hat{\varphi})$ isometrically onto $\mathcal{P}_{\varphi}$, i.e., if $g_{i}=\boldsymbol{P}_{\varphi} f_{i}$ with $f_{i} \in L^{2}(\mathbb{R} \mid \operatorname{supp} \hat{\varphi}), i=1,2$, then

$$
\left\langle g_{1}, g_{2}\right\rangle_{\varphi}=\left\langle f_{1}, f_{2}\right\rangle \text {. }
$$

Proof. Since the Fourier transform of $g_{i}=\boldsymbol{P}_{\varphi} f_{i}$ is $\hat{g}_{i}(\xi)=\sqrt{2 \pi} \overline{\hat{\varphi}(\xi)} \hat{f}_{i}(\xi), \xi \in$ $\mathbb{R}$, we have by Parseval's formula that $\left\langle g_{1}, g_{2}\right\rangle_{\varphi}=\left\langle\hat{f}_{1}, \hat{f}_{2}\right\rangle=\left\langle f_{1}, f_{2}\right\rangle$. If $g=$ $\boldsymbol{P}_{\varphi} f$ with $f \in L^{2}(\mathbb{R})$, then the function $f_{0}$ defined by $\hat{f}_{0}(\xi)=\hat{g}(\xi) /\left(\sqrt{2 \pi} \frac{\hat{\varphi}(\xi)}{}\right)$ in case $\xi \in \operatorname{supp} \hat{\varphi}$ and $\hat{f}_{0}(\xi)=0$ else is in $L^{2}(\mathbb{R} \mid \operatorname{supp} \hat{\varphi})$ and $g=\boldsymbol{P}_{\varphi} f_{0}$. Thus, $\boldsymbol{P}_{\varphi} L^{2}(\mathbb{R} \mid \operatorname{supp} \hat{\varphi})=\mathcal{P}_{\varphi}$. Because of the isometry relation (6), $\mathcal{P}_{\varphi}$ endowed with the inner product $\langle\cdot, \cdot\rangle_{\varphi}$ becomes a Hilbert space as is $L^{2}(\mathbb{R} \mid \operatorname{supp} \hat{\varphi})$ with respect to the inner product $\langle\cdot, \cdot\rangle$ of $L^{2}(\mathbb{R})$.

For any $x \in \mathbb{R}$ we define the function $\varphi_{x} \in L^{2}(\mathbb{R} \mid \operatorname{supp} \hat{\varphi})$ by $\varphi_{x}(y)=$ $\varphi(y-x), y \in \mathbb{R}$. Since $\left(\boldsymbol{P}_{\varphi} \varphi_{x}\right)(y)=\Phi(y-x)=K(x, y)$, we have $K(x, \cdot) \in \mathcal{P}_{\varphi}$. For any function $g=\boldsymbol{P}_{\varphi} f_{0}$ with $f_{0} \in L^{2}(\mathbb{R} \mid \operatorname{supp} \hat{\varphi})$ and any $x \in \mathbb{R}$ we now infer by means of (마) that

$$
\begin{aligned}
\langle g, K(x, \cdot)\rangle_{\varphi} & =\left\langle\boldsymbol{P}_{\varphi} f_{0}, \boldsymbol{P}_{\varphi} \varphi_{x}\right\rangle_{\varphi} \\
& =\left\langle f_{0}, \varphi_{x}\right\rangle \\
& =\int_{-\infty}^{\infty} f_{0}(y) \overline{\varphi(y-x)} d y \\
& =\left(\boldsymbol{P}_{\varphi} f_{0}\right)(x) \\
& =g(x) .
\end{aligned}
$$


Thus, $K(x, y)$ is the reproducing kernel in the Hilbert space $\mathcal{P}_{\varphi}$. This concludes the proof of Proposition 1.

We remark that Eq. (6) should not be taken as a definition of the inner product $\left\langle g_{1}, g_{2}\right\rangle_{\varphi}$, because it implies inversion of operator $\boldsymbol{P}_{\varphi}$ which, in general, is an ill-posed problem. We shall need the following subspaces of domain $L^{2}(\mathbb{R})$ and range $\mathcal{P}_{\varphi}$ of operator $\boldsymbol{P}_{\varphi}$.

Definition 1 The shift-invariant subspace $V_{\lambda}(\varphi) \subseteq L^{2}(\mathbb{R})$ is the closed linear span of $\left\{\varphi_{n}=\varphi(\cdot-n \lambda) ; n \in \mathbb{Z}\right\}$ in $L^{2}(\mathbb{R})$.

Definition 2 The subspace $\mathcal{R}_{\lambda}(\varphi) \subseteq \mathcal{P}_{\varphi}$ ("reconstruction subspace") is the closed linear span of $\left\{\Phi_{n}=\Phi(\cdot-n \lambda) ; n \in \mathbb{Z}\right\}$ in $\mathcal{P}_{\varphi}$.

Remark 1 The shift-invariant spaces $V_{T}(\varphi), T>0$, of Unser 28] are generated by translated and dilated versions $\varphi\left(\frac{x}{T}-n\right), n \in \mathbb{Z}$, of a generating function $\varphi$.

Proposition $2 \boldsymbol{P}_{\varphi}$ is an isometry from $V_{\lambda}(\varphi)$ onto $\mathcal{R}_{\lambda}(\varphi)$. If $\left\{\Phi_{n} ; n \in \mathbb{Z}\right\}$ forms a Riesz basis in $L^{2}(\mathbb{R})$, then $\mathcal{R}_{\lambda}(\varphi)$ may be identified with the shiftinvariant subspace $V_{\lambda}(\Phi) \subseteq L^{2}(\mathbb{R})$ defined as the closed linear span of $\left\{\Phi_{n} ; n \in\right.$ $\mathbb{Z}\}$ in $L^{2}(\mathbb{R})$.

Proof. Since $\boldsymbol{P}_{\varphi} \varphi_{n}=\Phi_{n} \forall n \in \mathbb{Z}$ and, by Proposition 1, $\boldsymbol{P}_{\varphi}$ is an isometry from $V_{\lambda}(\varphi) \subseteq L^{2}(\mathbb{R} \mid \operatorname{supp} \hat{\varphi})$ into $\mathcal{P}_{\varphi}$, it follows that $\boldsymbol{P}_{\varphi} V_{\lambda}(\varphi)=\mathcal{R}_{\lambda}(\varphi)$.

An element $v(x)=\sum_{n \in \mathbb{Z}}^{\text {finite }} c_{n} \Phi_{n}$ of the linear span of $\left\{\Phi_{n} ; n \in \mathbb{Z}\right\}$ has norms in $\mathcal{P}_{\varphi}$ and $L^{2}(\mathbb{R})$, resp.,

$$
\|v\|_{\varphi}=\left[\int_{0}^{1}|C(\xi)|^{2} A_{\varphi}(\Lambda \xi) d \xi\right]^{1 / 2},\|v\|=\left[\int_{0}^{1}|C(\xi)|^{2} A_{\Phi}(\Lambda \xi) d \xi\right]^{1 / 2},
$$

where $\Lambda=2 \pi / \lambda, C(\xi)=\sum_{n \in \mathbb{Z}}^{\text {finite }} c_{n} e^{-2 \pi i n \xi}, A_{\varphi}(\xi)$ is the central term in (4), and $A_{\Phi}(\xi)=\Lambda \sum_{k \in \mathbb{Z}}|\hat{\Phi}(\xi+k \Lambda)|^{2}$. Because of inequality (4) we have norm equivalence $\|v\|_{\varphi} \asymp\left[\int_{0}^{1}|C(\xi)|^{2} d \xi\right]^{1 / 2}$. If $\left\{\Phi_{n} ; n \in \mathbb{Z}\right\}$ also forms a Riesz basis, a similar inequality will hold for $A_{\Phi}(\xi)$, so that $\|v\| \asymp\left[\int_{0}^{1}|C(\xi)|^{2} d \xi\right]^{1 / 2}$. Then, $\|v\|_{\varphi} \asymp\|v\|$ and consequently $\mathcal{R}_{\lambda}(\varphi)=V_{\lambda}(\Phi)$, which concludes the proof of Proposition 2 ,

In case of a Gaussian function $\varphi,\left\{\Phi_{n} ; n \in \mathbb{Z}\right\}$ indeed forms a Riesz basis in $L^{2}(\mathbb{R})$ [17]. But examples can be given where, albeit $\varphi$ satisfies all the assumptions made in Section 2, we have

$$
0<A_{\Phi}(\xi) \leq \frac{1}{k} \text { a.e. } \xi \in S_{k}
$$

for some $\lambda \in \boldsymbol{\Lambda}$ and a sequence of subsets $S_{k} \subseteq \mathbb{R}, k=1,2, \ldots$, of positive measure. As a consequence, $\left\{\Phi_{n}=\Phi(\cdot-n \lambda) ; n \in \mathbb{Z}\right\}$ neither can form a Riesz basis nor a frame in $L^{2}(\mathbb{R})$, cf. [7, Theorem 7.2.3]. 
Proposition 3 Localization space $\mathcal{P}_{\varphi}$ has the orthogonal decomposition

$$
\mathcal{P}_{\varphi}=\mathcal{R}_{\lambda}(\varphi) \oplus \mathcal{N}_{\lambda}(\varphi)
$$

where subspace $\mathcal{N}_{\lambda}(\varphi)$ consists of all functions $h \in \mathcal{P}_{\varphi}$ with the property that $h(n \lambda)=0 \forall n \in \mathbb{Z}$. Every function $g \in \mathcal{R}_{\lambda}(\varphi)$ is completely determined by the sample values $g(n \lambda), n \in \mathbb{Z}$.

Proof. The space $L^{2}(\mathbb{R})$ has the orthogonal decomposition

$$
L^{2}(\mathbb{R})=V_{\lambda}(\varphi) \oplus V_{\lambda}(\varphi)^{\perp}
$$

Application of $\boldsymbol{P}_{\varphi}$ yields $\mathcal{P}_{\varphi}=\boldsymbol{P}_{\varphi} V_{\lambda}(\varphi)+\boldsymbol{P}_{\varphi}\left(V_{\lambda}(\varphi)^{\perp}\right)$. By Proposition 2, $\boldsymbol{P}_{\varphi} V_{\lambda}(\varphi)=\mathcal{R}_{\lambda}(\varphi)$. We now show that $\boldsymbol{P}_{\varphi}\left(V_{\lambda}(\varphi)^{\perp}\right)=\mathcal{N}_{\lambda}(\varphi)$. If $f \in V_{\lambda}(\varphi)^{\perp}$, then $h=\boldsymbol{P}_{\varphi} f$ satisfies

$$
h(n \lambda)=\left(\boldsymbol{P}_{\varphi} f\right)(n \lambda)=\langle f, \varphi(\cdot-n \lambda)\rangle=0 \quad \forall n \in \mathbb{Z},
$$

So, $h \in \mathcal{N}_{\lambda}(\varphi)$. Conversely, if $h \in \mathcal{N}_{\lambda}(\varphi)$, then $h=\boldsymbol{P}_{\varphi} f$ for some $f \in L^{2}(\mathbb{R})$. Reading (9) from the left to the right shows that $f$ is orthogonal on the dense subset $\left\{\varphi_{n} ; n \in \mathbb{Z}\right\}$ of $V_{\lambda}(\varphi)$. So, $f \in V_{\lambda}(\varphi)^{\perp}$.

The orthogonality $\mathcal{R}_{\lambda}(\varphi) \perp \mathcal{N}_{\lambda}(\varphi)$ in $\mathcal{P}_{\varphi}$ (implying $\mathcal{R}_{\lambda}(\varphi) \cap \mathcal{N}_{\lambda}(\varphi)=\{0\}$ ) is seen as follows. If $g \in \mathcal{R}_{\lambda}(\varphi), h \in \mathcal{N}_{\lambda}(\varphi)$, then $g=\boldsymbol{P}_{\varphi} f_{1}$ for some $f_{1} \in$ $V_{\lambda}(\varphi) \subseteq L^{2}(\mathbb{R} \mid \operatorname{supp} \hat{\varphi})$ and $h=\boldsymbol{P}_{\varphi} f_{2}$ for some $f_{2} \in V_{\lambda}(\varphi)^{\perp}$. Define $f_{0} \in$ $L^{2}(\mathbb{R} \mid \operatorname{supp} \hat{\varphi})$ by $\hat{f}_{0}=\chi_{\operatorname{supp} \hat{\varphi}} \cdot \hat{f}_{2}$. Then $\boldsymbol{P}_{\varphi} f_{2}=\boldsymbol{P}_{\varphi} f_{0}$, So, by Proposition 1 , we have $\langle g, h\rangle_{\varphi}=\left\langle\boldsymbol{P}_{\varphi} f_{1}, \boldsymbol{P}_{\varphi} f_{0}\right\rangle_{\varphi}=\left\langle f_{1}, f_{0}\right\rangle=\left\langle\hat{f}_{1}, \hat{f}_{0}\right\rangle=\left\langle\hat{f}_{1}, \hat{f}_{2}\right\rangle=\left\langle f_{1}, f_{2}\right\rangle=$ 0. Thus, $g \perp h$ in $\mathcal{P}_{\varphi}$.

Finally, if $g_{1}, g_{2} \in \mathcal{R}_{\lambda}(\varphi)$ with $g_{1}(n \lambda)=g_{2}(n \lambda) \forall n \in \mathbb{Z}$, then $g_{1}-g_{2} \in$ $\mathcal{R}_{\lambda}(\varphi) \cap \mathcal{N}_{\lambda}(\varphi)=\{0\}$. Hence, $g_{1}=g_{2}$, which concludes the proof of Proposition 3 ,

We refer to [9], 10], 11] where orthogonal decompositons of Hilbert spaces have been used for the purpose of sampling and reconstruction in a more general and abstract setting.

\section{Perfect Reconstruction in a Subspace}

Since $\left\{\Phi_{n} ; n \in \mathbb{Z}\right\}$ not always forms a Riesz basis, the proof of the next theorem is based on frame theory. Concerning frame theory we refer to [7], [15], [31. Because $\left\{\Phi_{n} ; n \in \mathbb{Z}\right\}$ also not always forms a frame for its closed linear span in $L^{2}(\mathbb{R})$ we cannot resort to, e.g., [25, Theorem 2.1]. The RKHS property of $\mathcal{P}_{\varphi}$ will now prove helpful; see [20], [22], 30] with regard to application of reproducing kernel Hilbert spaces in sampling theory. 
Theorem 1 Let the prefilter function $\varphi$ and the set $\boldsymbol{\Lambda} \subseteq \mathbb{R}_{+}$be as assumed in Section 2 and let $\lambda \in \boldsymbol{\Lambda}$. Then any function $g \in \mathcal{R}_{\lambda}(\varphi)$ can be perfectly reconstructed from its sample values $g(n \lambda), n \in \mathbb{Z}$, by the series

$$
g(x)=\sum_{n=-\infty}^{\infty} g(n \lambda) \Phi_{\text {int }}(x-n \lambda), x \in \mathbb{R},
$$

where the interpolating function $\Phi_{\mathrm{int}} \in \mathcal{R}_{\lambda}(\varphi)$ is given by

$$
\hat{\Phi}_{\text {int }}(\xi)=\frac{\hat{\Phi}(\xi)}{\frac{\Lambda}{\sqrt{2 \pi}} \sum_{n \in \mathbb{Z}} \hat{\Phi}(\xi+n \Lambda)}, \Lambda=\frac{2 \pi}{\lambda} .
$$

The series in (10) converges in the norm of $\mathcal{P}_{\varphi}$ and uniformly on $\mathbb{R}$.

Proof. Since $\left\{\varphi_{n} ; n \in \mathbb{Z}\right\}$ is a Riesz basis of $V_{\lambda}(\varphi)$ it also forms a frame for that space. Hence, there exist positive constants $A, B$ (actually the same as in (44)) so that for all $f \in V_{\lambda}(\varphi)$ we have

$$
A\|f\|^{2} \leq \sum_{n \in \mathbb{Z}}\left|\left\langle f, \varphi_{n}\right\rangle\right|^{2} \leq B\|f\|^{2} .
$$

Because $\Phi_{n}=\boldsymbol{P}_{\varphi} \varphi_{n}$ and $\boldsymbol{P}_{\varphi}: V_{\lambda}(\varphi) \rightarrow \mathcal{R}_{\lambda}(\varphi)$ is an isometry we conclude that for all $g \in \mathcal{R}_{\lambda}(\varphi)$ it holds that

$$
A\|g\|_{\varphi}^{2} \leq \sum_{n \in \mathbb{Z}}\left|\left\langle g, \Phi_{n}\right\rangle_{\varphi}\right|^{2} \leq B\|g\|_{\varphi}^{2}
$$

Thus, $\left\{\Phi_{n} ; n \in \mathbb{Z}\right\}$ is a frame for the Hilbert space $\mathcal{R}_{\lambda}(\varphi) \subseteq \mathcal{P}_{\varphi}$. The corresponding frame operator $S: \mathcal{R}_{\lambda}(\varphi) \rightarrow \mathcal{R}_{\lambda}(\varphi)$ has the representation $S g=\sum_{n \in \mathbb{Z}}\left\langle g, \Phi_{n}\right\rangle_{\varphi} \Phi_{n}$. By frame theory it has a continuous inverse $S^{-1}$ : $\mathcal{R}_{\lambda}(\varphi) \rightarrow \mathcal{R}_{\lambda}(\varphi)$ yielding the reconstruction formula

$$
g=\sum_{n \in \mathbb{Z}}\left\langle g, \Phi_{n}\right\rangle_{\varphi} S^{-1} \Phi_{n}
$$

Because of the RKHS property of the localization space $\mathcal{P}_{\varphi}$ we already know that $\left\langle g, \Phi_{n}\right\rangle_{\varphi}=\langle g, \Phi(\cdot-n \lambda)\rangle_{\varphi}=g(n \lambda)$. The uniquely determined solution $\varphi_{\text {dual }} \in V_{\lambda}(\varphi)$ of the system of equations

$$
\left\langle\varphi_{\text {dual }}, \varphi(\cdot-n \lambda)\right\rangle=\delta_{n}, n \in \mathbb{Z},
$$

exists in virtue of the positive lower bound in Ineq. (4) and has Fourier transform

$$
\hat{\varphi}_{\text {dual }}(\xi)=\frac{\hat{\varphi}(\xi)}{\Lambda \sum_{k \in \mathbb{Z}}|\hat{\varphi}(\xi+k \Lambda)|^{2}} .
$$


(The argument in [17] in case of a Gaussian function $\varphi$ carries over to the general case without changes.) Defining $\Phi_{\text {int }}=\boldsymbol{P}_{\varphi} \varphi_{\text {dual }}$ we infer, again by isometry, that

$$
\begin{aligned}
S\left[\Phi_{\text {int }}(\cdot-m \lambda)\right] & =\sum_{n \in \mathbb{Z}}\left\langle\Phi_{\text {int }}(\cdot-m \lambda), \Phi_{n}\right\rangle_{\varphi} \Phi_{n} \\
& =\sum_{n \in \mathbb{Z}}\left\langle\boldsymbol{P}_{\varphi}\left(\varphi_{\text {dual }}(\cdot-m \lambda)\right), \boldsymbol{P}_{\varphi} \varphi_{n}\right\rangle_{\varphi} \Phi_{n} \\
& =\sum_{n \in \mathbb{Z}}\left\langle\varphi_{\text {dual }}(\cdot-m \lambda), \varphi(\cdot-n \lambda)\right\rangle \Phi_{n} \\
& =\sum_{n \in \mathbb{Z}} \delta_{m n} \Phi_{n} \\
& =\Phi_{m} .
\end{aligned}
$$

Thus, $S^{-1} \Phi_{m}=\Phi_{\text {int }}(\cdot-m \lambda)$ and (12) turns into (10). By means of the Fourier domain representation of $\boldsymbol{P}_{\varphi}$ we readily obtain

$$
\Phi_{\mathrm{int}}(x)=\frac{1}{\sqrt{2 \pi}} \int_{-\infty}^{\infty} e^{i x \xi} \frac{|\hat{\varphi}(\xi)|^{2}}{\frac{\Lambda}{\sqrt{2 \pi}} \sum_{n \in \mathbb{Z}}|\hat{\varphi}(\xi+n \Lambda)|^{2}} d \xi,
$$

which proves (11).

Frame theory ensures convergence of the series in (12) in the norm $\|\cdot\|_{\varphi}$. Let $g_{N}$ be the $N$ th partial sum. Since $\left(g-g_{N}\right)(x)=\left\langle g-g_{N}, \Phi(\cdot-x)\right\rangle_{\varphi}$, application of the Cauchy-Schwarz inequality results for any $x \in \mathbb{R}$ in the estimate

$$
\left|g(x)-g_{N}(x)\right| \leq\left\|g-g_{N}\right\|_{\varphi}\|\Phi(\cdot-x)\|_{\varphi}=\|\varphi\|\left\|g-g_{N}\right\|_{\varphi},
$$

which proves uniform convergence $g_{N} \rightarrow g$ on $\mathbb{R}$ and concludes the proof of Theorem 1.

We note that because of $\Phi_{\text {int }}(n \lambda)=\left\langle\varphi_{\text {dual }}, \varphi(\cdot-n \lambda)\right\rangle$ the interpolating function $\Phi_{\text {int }}$ has the interpolation property

$$
\Phi_{\text {int }}(n \lambda)=\delta_{n}, n \in \mathbb{Z} .
$$

Remark 2 A similar result holds for the space $V_{\lambda}(\varphi)$ : Every $f \in V_{\lambda}(\varphi)$ can be perfectly reconstructed from its sample values $f(n \lambda), n \in \mathbb{Z}$, by the series

$$
f(x)=\sum_{n=-\infty}^{\infty} f(n \lambda) \varphi_{\mathrm{int}}(x-n \lambda),
$$

where the interpolating function $\varphi_{\mathrm{int}} \in V_{\lambda}(\varphi)$ is given by

$$
\hat{\varphi}_{\text {int }}(\xi)=\frac{\hat{\varphi}(\xi)}{\frac{\Lambda}{\sqrt{2 \pi}} \sum_{n \in \mathbb{Z}} \hat{\varphi}(\xi+n \Lambda)}, \Lambda=\frac{2 \pi}{\lambda},
$$


provided that the denominator in (15) does not vanish (and some additional regularity assumptions on $\varphi$ ). This result is due to Walter [30] who proved it for orthonormal bases $\{\varphi(\cdot-n) ; n \in \mathbb{Z}\}$. The argument is essentially the same for Riesz bases, see [17] for the case of a Gaussian function $\varphi$. We shall return to this topic at the end of Section 6.

\section{Error Estimate}

If $g$ is in $\mathcal{P}_{\varphi} \backslash \mathcal{R}_{\lambda}(\varphi)$, then the series in Theorem 1 still may be computed but will result in an orthogonal projection $\tilde{g}$ of $g$ onto $\mathcal{R}_{\lambda}(\varphi)$ rather than in $g$ itself. The purpose of the present section is to estimate the error $|g(x)-\tilde{g}(x)|$ for any $x \in \mathbb{R}$.

Let $g=\boldsymbol{P}_{\varphi} f$ with $f \in L^{2}(\mathbb{R})$ and define $\tilde{f}=P_{\lambda} f$ where $P_{\lambda}$ is the orthogonal projection from $L^{2}(\mathbb{R})$ onto $V_{\lambda}(\varphi)$. Then $\tilde{g}=\boldsymbol{P}_{\varphi} \tilde{f}=\boldsymbol{P}_{\varphi} P_{\lambda} f$. So, we need to estimate $\left|\left(\boldsymbol{P}_{\varphi} f\right)(x)-\left(\boldsymbol{P}_{\varphi} P_{\lambda} f\right)(x)\right|$ for arbitrary $f \in L^{2}(\mathbb{R})$ and $x \in \mathbb{R}$.

Proposition 4 Let $\lambda \in \boldsymbol{\Lambda}$. The orthogonal projection $P_{\lambda}: L^{2}(\mathbb{R}) \rightarrow V_{\lambda}(\varphi)$ is given by

$$
\widehat{\left(P_{\lambda} f\right)}(\xi)=\left(\Lambda \sum_{n \in \mathbb{Z}} \hat{f}(\xi+n \Lambda) \overline{\hat{\varphi}(\xi+n \Lambda)}\right) \hat{\varphi}_{\text {dual }}(\xi),
$$

where $\Lambda=2 \pi / \lambda$ and $\hat{\varphi}_{\text {dual }}$ is as in (13).

Proof. In the special case $\lambda=1$ representation (16) is readily obtained from 4, Theorem 2.9]. The general case $\lambda \in \boldsymbol{\Lambda}$ can then be deduced from the previous one by rescaling. This concludes the proof of Proposition 4 .

Proposition 5 Let $\lambda \in \boldsymbol{\Lambda}$. The operator $\boldsymbol{Q}_{\lambda}=\boldsymbol{P}_{\varphi} P_{\lambda}: L^{2}(\mathbb{R}) \rightarrow \mathcal{R}_{\lambda}(\varphi)$ is given by

$$
\left(\boldsymbol{Q}_{\lambda} f\right)(x)=\int_{-\infty}^{\infty} Q_{\lambda}(x, \xi) \hat{f}(\xi) \overline{\hat{\varphi}(\xi)} d \xi, x \in \mathbb{R}
$$

where

$$
Q_{\lambda}(x, \xi)=\frac{\sum_{n \in \mathbb{Z}} e^{i x(\xi+n \Lambda)}|\hat{\varphi}(\xi+n \Lambda)|^{2}}{\sum_{n \in \mathbb{Z}}|\hat{\varphi}(\xi+n \Lambda)|^{2}} .
$$

Proof. Let $f \in \mathcal{S}(\mathbb{R})$ where $\mathcal{S}(\mathbb{R})$ is the Schwartz space of $C^{\infty}$ functions on $\mathbb{R}$ 
rapidly decaying at infinity. By (11), (13) and (16) we get

$$
\begin{aligned}
\left(\boldsymbol{P}_{\varphi} P_{\lambda} f\right)(x) & =\int_{-\infty}^{\infty} e^{i x \xi} \overline{\hat{\varphi}(\xi)} \widehat{\left(P_{\lambda} f\right)}(\xi) d \xi \\
& =\int_{-\infty}^{\infty} e^{i x \xi} \overline{\hat{\varphi}(\xi)}\left\{\left(\Lambda \sum_{n \in \mathbb{Z}} \hat{f}(\xi+n \Lambda) \overline{\hat{\varphi}(\xi+n \Lambda)}\right) \hat{\varphi}_{\text {dual }}(\xi)\right\} d \xi \\
& =\Lambda \sum_{n \in \mathbb{Z}} \int_{-\infty}^{\infty} e^{i x(\xi-n \Lambda)} \overline{\hat{\varphi}(\xi)} \hat{f}(\xi) \frac{|\hat{\varphi}(\xi-n \Lambda)|^{2}}{\Lambda \sum_{k \in \mathbf{Z}}|\hat{\varphi}(\xi+k \Lambda)|^{2}} d \xi \\
& =\int_{-\infty}^{\infty} Q_{\lambda}(x, \xi) \hat{f}(\xi) \overline{\hat{\varphi}(\xi)} d \xi .
\end{aligned}
$$

Since $P_{\lambda}$ and $\boldsymbol{P}_{\varphi}$ are continuous operators from $L^{2}(\mathbb{R})$ into itself, so is $\boldsymbol{Q}_{\lambda}$. Consequently, the representation (17) extends to all of $L^{2}(\mathbb{R})$ by continuity. This completes the proof of Proposition 5.

Lemma 1 (Generalized Chebyshev Inequality) Let $\phi$ be a probability density function on $\mathbb{R}$, i.e., $\phi(x) \geq 0, \int_{\mathbb{R}} \phi(x) d x$ exists and is equal to 1 . Suppose that $M=\int_{\mathbb{R}} w(|x|) \phi(x) d x<\infty$, where $w(x)$ for $x>0$ is positive and monotonically increasing. Then

$$
\int_{|x| \geq t} \phi(x) d x \leq \frac{M}{w(t)} \forall t>0 .
$$

Proof. Let $t>0$. Then

$$
\begin{aligned}
\int_{|x| \geq t} \phi(x) d x & =\int_{|x| \geq t} \frac{w(|x|) \phi(x)}{w(|x|)} d x \\
& \leq \frac{1}{w(t)} \int_{|x| \geq t} w(|x|) \phi(x) d x \\
& \leq \frac{1}{w(t)} \int_{\mathbb{R}} w(|x|) \phi(x) d x \\
& =\frac{M}{w(t)}
\end{aligned}
$$

which proves Lemma 1. We found the preceding theorem as an exercise in the textbook [13. Note that the normalization condition $\int_{\mathbb{R}} \phi(x) d x=1$ is unnecessary.

Theorem 2 Let $f \in L^{2}(\mathbb{R}), g=\boldsymbol{P}_{\varphi} f$ and $\tilde{g}=\boldsymbol{Q}_{\lambda} f$, where $\lambda \in \boldsymbol{\Lambda}$. Then for all $x \in \mathbb{R}$ it holds that

$$
|g(x)-\tilde{g}(x)|^{2} \leq 8 M_{w}(\varphi)\left(\sum_{n=1}^{\infty} \frac{1}{w((2 n-1) \pi / \lambda)}\right)\|g\|_{\varphi}^{2} .
$$


Proof. By (11) and (17) we have

$$
g(x)-\tilde{g}(x)=\int_{-\infty}^{\infty}\left(e^{i x \xi}-Q_{\lambda}(x, \xi)\right) \hat{f}(\xi) \overline{\hat{\varphi}(\xi)} d \xi
$$

Since $\left|e^{i x \xi}-Q_{\lambda}(x, \xi)\right| \leq 2 E_{\lambda}(\xi)$, where

$$
E_{\lambda}(\xi)=\frac{\sum_{n \in \mathbb{Z} \backslash\{0\}}|\hat{\varphi}(\xi+n \Lambda)|^{2}}{\sum_{n \in \mathbb{Z}}|\hat{\varphi}(\xi+n \Lambda)|^{2}},
$$

we obtain by means of the Cauchy-Schwarz inequality that

$$
|g(x)-\tilde{g}(x)|^{2} \leq 4\left(\int_{-\infty}^{\infty} E_{\lambda}(\xi)|\hat{f}(\xi)|^{2} d \xi\right)\left(\int_{-\infty}^{\infty} E_{\lambda}(\xi)|\hat{\varphi}(\xi)|^{2} d \xi\right) .
$$

Because

$$
0 \leq E_{\lambda}(\xi) \leq 1
$$

we get for the first integral factor $\int_{\mathbb{R}} E_{\lambda}(\xi)|\hat{f}(\xi)|^{2} d \xi \leq\|f\|^{2}$. Writing

$$
\int_{-\infty}^{\infty} E_{\lambda}(\xi)|\hat{\varphi}(\xi)|^{2} d \xi=I(0, \pi / \lambda)+I(\pi / \lambda, \infty)
$$

where we have used the notation

$$
I(a, b)=\int_{a \leq|\xi|<b} E_{\lambda}(\xi)|\hat{\varphi}(\xi)|^{2} d \xi,
$$

we need to estimate the two constituent integrals. This will be done by means of inequalities (18) and (20).

Integral $I(\pi / \lambda, \infty)$ :

$$
I(\pi / \lambda, \infty) \leq \int_{|\xi| \geq \pi / \lambda}|\hat{\varphi}(\xi)|^{2} d \xi \leq \frac{M_{w}(\varphi)}{w(\pi / \lambda)} .
$$


Integral $I(0, \pi / \lambda)$ :

$$
\begin{aligned}
I(0, \pi / \lambda) & =\int_{-\pi / \lambda}^{\pi / \lambda}\left(1-E_{\lambda}(\xi)\right)\left(\sum_{n \in \mathbb{Z} \backslash\{0\}}|\hat{\varphi}(\xi+n \Lambda)|^{2}\right) d \xi \\
& \leq \sum_{n=1}^{\infty}\left\{\int_{-\pi / \lambda}^{\pi / \lambda}|\hat{\varphi}(\xi+n \Lambda)|^{2} d \xi+\int_{-\pi / \lambda}^{\pi / \lambda}|\hat{\varphi}(\xi-n \Lambda)|^{2} d \xi\right\} \\
& \leq \sum_{n=1}^{\infty}\left\{\int_{-\pi / \lambda}^{\infty}|\hat{\varphi}(\xi+n \Lambda)|^{2} d \xi+\int_{-\infty}^{\pi / \lambda}|\hat{\varphi}(\xi-n \Lambda)|^{2} d \xi\right\} \\
& =\sum_{n=1}^{\infty}\left\{\int_{(2 n-1) \pi / \lambda}^{\infty}|\hat{\varphi}(\xi)|^{2} d \xi+\int_{-\infty}^{(-2 n+1) \pi / \lambda}|\hat{\varphi}(\xi)|^{2} d \xi\right\} \\
& =\sum_{n=1}^{\infty} \int_{|\xi| \geq(2 n-1) \pi / \lambda}|\hat{\varphi}(\xi)|^{2} d \xi \\
& \leq \sum_{n=1}^{\infty} \frac{M_{w}(\varphi)}{w((2 n-1) \pi / \lambda)} .
\end{aligned}
$$

Because of growth condition (3) imposed on the weight function $w$, the infinite series converges. By (21), (22) it follows that

$$
\int_{-\infty}^{\infty} E_{\lambda}(\xi)|\hat{\varphi}(\xi)|^{2} d \xi \leq 2 M_{w}(\varphi) \sum_{n=1}^{\infty} \frac{1}{w((2 n-1) \pi / \lambda)} .
$$

Consequently,

$$
|g(x)-\tilde{g}(x)|^{2} \leq 8 M_{w}(\varphi)\left(\sum_{n=1}^{\infty} \frac{1}{w((2 n-1) \pi / \lambda)}\right)\|f\|^{2} .
$$

By Proposition 1 we may assume that $f \in L^{2}(\mathbb{R} \mid \operatorname{supp} \hat{\varphi})$. Then $\|f\|=\|g\|_{\varphi}$, which concludes the proof of Theorem 2 .

\subsection{Example: Monomial Weight $w(\xi)=|\xi|^{s}, s>1$}

We define

$$
\mu_{s}(\varphi)=\left[\frac{M_{w}(\varphi)}{\|\varphi\|^{2}}\right]^{\frac{1}{s}}
$$

It is not difficult to prove the identity $\sum_{n=1}^{\infty}(2 n-1)^{-s}=\left(1-2^{-s}\right) \zeta(s)$ for the Riemann zeta function $\zeta(s)=\sum_{n=1}^{\infty} n^{-s}, \Re(s)>0$. Then, Ineq. (19) turns into

$$
|g(x)-\tilde{g}(x)|^{2} \leq 8\left(1-2^{-s}\right) \zeta(s)\left[\frac{\mu_{s}(\varphi) \lambda}{\pi}\right]^{s}\|\varphi\|^{2}\|g\|_{\varphi}^{2} .
$$


We observe that the right-hand side of (23) decays order of $s$ to 0 as

$$
0 \leftarrow \lambda<\lambda_{0}=\frac{\pi}{\mu_{s}(\varphi)}
$$

Here, of course, inf $\boldsymbol{\Lambda}=0$ is supposed. Note that the upper bound in (24) becomes more and more tight as $s \rightarrow \infty$. In the special case $s=2$ we obtain by means of the celebrated identity $\zeta(2)=\pi^{2} / 6$ of Eule1 1$]$ the remarkably simple inequality

$$
|g(x)-\tilde{g}(x)|^{2} \leq\left(\mu_{2}(\varphi) \lambda\right)^{2}\|\varphi\|^{2}\|g\|_{\varphi}^{2} .
$$

\section{Sampling Theorem}

The following theorem is a consequence of Theorem 1, Theorem 2 and the interpolation property (14).

Theorem 3 (Generalized Sampling Theorem) Let the prefilter $\boldsymbol{P}_{\varphi}$ and the set $\boldsymbol{\Lambda}$ of admissable sampling intervals be as assumed in Section 2. For arbitrary $\lambda \in \boldsymbol{\Lambda}$ the interpolating function $\Phi_{\text {int }} \in \mathcal{R}_{\lambda}(\varphi) \subseteq \mathcal{P}_{\varphi}$ is defined by (11). Then for any signal $g$ in the localization space $\mathcal{P}_{\varphi}$ of $\boldsymbol{P}_{\varphi}$ the series

$$
\tilde{g}(x)=\sum_{n=-\infty}^{\infty} g(n \lambda) \Phi_{\text {int }}(x-n \lambda)
$$

converges in the norm of $\mathcal{P}_{\varphi}$ and uniformly on $\mathbb{R}$ to a function $\tilde{g} \in \mathcal{R}_{\lambda}(\varphi)$ with the property that $\tilde{g}(n \lambda)=g(n \lambda) \forall n \in \mathbb{Z}$. For all other $x \in \mathbb{R}$ the relative approximation error $\epsilon_{\lambda}(x)=|g(x)-\tilde{g}(x)| /\|g\|_{\varphi}$ satisfies the estimate

$$
\epsilon_{\lambda}^{2}(x) \leq 8 M_{w}(\varphi) \sum_{n=1}^{\infty} \frac{1}{w((2 n-1) \pi / \lambda)} .
$$

Theorem 3 may serve as pattern for the proof of specific sampling theorems. Examples are given in the following subsection.

Special attention deserves the upper bound in Ineq. (27). As seen in Section 5.1, it may allow for the identification of a critical sampling interval $\lambda_{0}$ with the property that the error $\epsilon_{\lambda}(x)$ diminishes substantially as soon as the sampling interval $\lambda$ falls under $\lambda_{0}$. Similarly to the Nyquist interval in the classical sampling theorem [24], 29], we may also get a link between $\lambda_{0}$ and some measure of bandwidth for the prefilter function $\varphi$ (or the prefilter $\boldsymbol{P}_{\varphi}$ ).

\footnotetext{
${ }^{1}$ See M. du Sautoy, The Music of Primes. Why an Unsolved Problem in Mathematics Matters, Fourth Estate, London, 2003, for a historical account
} 
Indeed, inequality (25) suggests the critical sampling interval $\lambda_{0}=1 / \sigma(\varphi)$, where $\sigma(\varphi)=\mu_{2}(\varphi)$ is defined by

$$
\sigma^{2}(\varphi)=\|\varphi\|^{-2} \int_{-\infty}^{\infty} \xi^{2}|\hat{\varphi}(\xi)|^{2} d \xi
$$

$\sigma(\varphi)$ may be viewed as the standard deviation of the probability density function $\|\hat{\varphi}\|^{-2}|\hat{\varphi}(\cdot)|^{2}$ in the frequency domain. Following Gabor 14 where a centered version of $\sigma(\varphi)$ called "effective frequency width" has been defined, we might take $\sigma(\varphi)$ as a measure of "soft bandwidth" for $\varphi$ (or $\boldsymbol{P}_{\varphi}$ ). Inequality (25) then tells us that, under the assumption $\sigma(\varphi)<\infty$, the sampling interval should always be chosen smaller than the reciprocal "soft bandwidth" $\sigma(\varphi)$.

A definition of "soft bandwidth" could, of course, also be based on other weights $w$ leading to, e.g., the moment-like quantities $\mu_{s}(\varphi)$ of Section 5.1.

\subsection{Instances of the Generalized Sampling Theorem}

1) The prefilter function

$$
\varphi(x)=\operatorname{sinc}(\pi \beta x) \stackrel{\text { Fourier }}{\longleftrightarrow} \hat{\varphi}(\xi)=\frac{1}{\sqrt{2 \pi} \beta} \chi_{[-\pi \beta, \pi \beta]}(\xi),
$$

where $\operatorname{sinc} x=(\sin x) / x$, defines the ideal low-pass filter of (two-sided) bandwidth $2 \pi \beta$. For an arbitrary weight function $w_{s}(\xi)=s(\pi \beta)^{1-s}|\xi|^{s-1}$ with $s>2$ we compute that $M_{w_{s}}(\varphi)=1 / \beta$. The Riesz basis condition (4) is fulfilled for $\lambda \in \boldsymbol{\Lambda}=[1 / \beta, \infty)$. Localization space $\mathcal{P}_{\varphi}$ coincides with the space of finiteenergy signals bandlimited to $[-\pi \beta, \pi \beta]$. With the special choice $\lambda=1 / \beta$ (Nyquist interval) we now obtain by means of Theorem 3 for fixed $g \in \mathcal{P}_{\varphi}$ and an arbitrary weight function $w_{s}(\xi), s>2$, observing that $\|g\|_{\varphi}=\beta\|g\|$, the inequality

$$
|g(x)-\tilde{g}(x)|^{2} \leq \frac{8 \beta}{s}\left(1-2^{-s+1}\right) \zeta(s-1)\|g\|^{2} .
$$

Since the upper bound tends to 0 as $s \rightarrow \infty$, it follows that $\tilde{g}(x)=g(x) \forall x \in \mathbb{R}$. Furthermore, by (11) we immediately see that $\Phi_{\text {int }}(x)=\operatorname{sinc}(\pi \beta x)$. As a result, we have proved the classical sampling theorem [24] showing that it is within the scope of our generalized approach.

2) For the prefilter function

$$
\varphi(x)=\frac{1}{\sqrt{2 \pi}(1 / \beta)} e^{-\frac{x^{2}}{2(1 / \beta)^{2}}} \stackrel{\text { Fourier }}{\longleftrightarrow} \hat{\varphi}(\xi)=\frac{1}{\sqrt{2 \pi}} e^{-\frac{\xi^{2}}{2 \beta^{2}}},
$$

a Gaussian probability density function of standard deviation $1 / \beta, \beta>0$, the weight function

$$
w(\xi)=e^{\frac{\xi^{2}}{2 \beta^{2}}}
$$


and $\boldsymbol{\Lambda}=\mathbb{R}_{+}$may be used. Since $M_{w}(\varphi)=\beta / \sqrt{2 \pi}<\infty$ and for $\lambda>0$ small enough (condition (32), see below, is sufficient)

$$
\sum_{n=1}^{\infty} \frac{1}{w((2 n-1) \pi / \lambda)}=\sum_{n=1}^{\infty} e^{-\frac{(\pi / \lambda)^{2}}{2\left(\frac{\beta}{2 n-1}\right)^{2}}}<2 e^{-\frac{(\pi / \lambda)^{2}}{2 \beta^{2}}}
$$

we obtain by Theorem 3 the estimate

$$
\left[|g(x)-\tilde{g}(x)| /\|g\|_{\varphi}\right]^{2} \leq \frac{16 \beta}{\sqrt{2 \pi}} e^{-\frac{(\pi / \lambda)^{2}}{2 \beta^{2}}} \forall x \in \mathbb{R} .
$$

Because of the "three-sigma rule" for the Gaussian function in the interpretation given in [16], the right-hand side of Ineq. (31) becomes small as soon as $\pi / \lambda>\pi \beta$ or, equivalently,

$$
0<\lambda<\lambda_{0}=\frac{1}{\beta}
$$

then decaying super-exponentially to 0 as $\lambda \rightarrow 0$. (By means of a specific inequality for the tails of the Gaussian function it is shown in [16] that the error becomes very small already when $\lambda=\lambda_{0}=1 / \beta$.) We note that $\lambda_{0}$ is related to the "soft bandwidth" $\sigma(\varphi)$ of the Gaussian function (30) by the equation $\lambda_{0}=1 /(\sqrt{2} \sigma(\varphi))$. The corresponding interpolating function $\Phi_{\text {int }}$ has been calculated in [16], [17].

3) The centered B-spline of order $m$ [27], $m=2,3, \ldots$,

$$
\begin{aligned}
\beta^{m-1}(x) & =(\underbrace{\beta^{0} * \ldots * \beta^{0}}_{m \text { times }})(x), \beta^{0}(x)=\chi_{\left[-\frac{1}{2}, \frac{1}{2}\right]}(x) \\
& \stackrel{\text { Fourier }}{\longleftrightarrow} \hat{\beta}^{m-1}(\xi)=\frac{1}{\sqrt{2 \pi}}\left(\frac{\sin (\xi / 2)}{\xi / 2}\right)^{m},
\end{aligned}
$$

may also be used as prefilter function $\varphi$. Any weight function

$$
w(\xi)=|\xi|^{s}, 1<s<2 m-1,
$$

may be taken. Since the central term in (44) has zeros when $\lambda=\frac{1}{2}, \frac{1}{3}, \frac{1}{4}, \ldots$, we choose $\boldsymbol{\Lambda}=\mathbb{R}_{+} \backslash\left\{\frac{1}{2}, \frac{1}{3}, \frac{1}{4}, \ldots\right\}$ as the set of admissable sampling intervals. Then Theorem 3 applies and Ineq. (23) becomes

$$
|g(x)-\tilde{g}(x)|^{2} \leq C \lambda^{s} \quad \forall x \in \mathbb{R},
$$

where $C$ is some finite constant not depending on $\lambda \in \boldsymbol{\Lambda}$. Now, let $\boldsymbol{\Lambda} \ni$ $\lambda \rightarrow \lambda_{\ell}=1 / \ell$ for $\ell \in\{2,3, \ldots\}$ held constant. Since zeros arising in (11) in the denominator are cancelled by corresponding zeros of the numerator 
$\hat{\Phi}(\xi)=\hat{\beta}^{2 m-1}(\xi)$ we observe convergence $\Phi_{\text {int }} \rightarrow \Phi_{\ell \text {,int }}$ in $L^{2}(\mathbb{R})$ where $\Phi_{\ell \text {,int }}$ is given by

$$
\hat{\Phi}_{\ell, \text { int }}(\xi)=\frac{\hat{\Phi}_{\ell}(\xi)}{\frac{\Lambda_{\ell}}{\sqrt{2 \pi}} \sum_{n \in \mathbb{Z}} \hat{\Phi}_{\ell}\left(\xi+n \Lambda_{\ell}\right)}, \Lambda_{\ell}=\frac{2 \pi}{\lambda_{\ell}},
$$

with $\hat{\Phi}_{\ell}(\xi)=(1 / \ell) \hat{\Phi}(\xi / \ell)$. In Figure $1(\mathrm{a})-(\mathrm{d})$ this bevaviour is depicted for prefilter function $\varphi(x)=\beta^{2}(x)$ in case $\ell=4$.

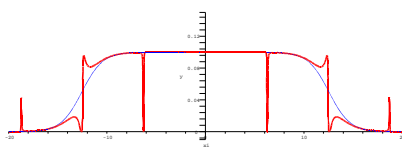

(a)

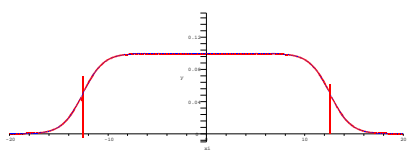

(c)

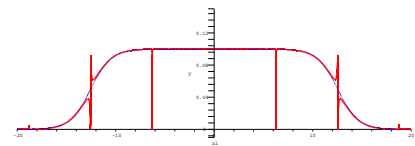

(b)

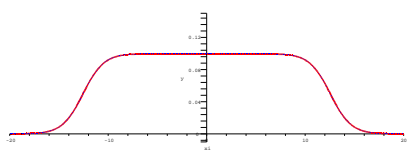

(d)

Fig. 1. $\Phi_{\ell \text {,int }}(\ell=4)$ and $\Phi_{\text {int }}$ as $\lambda \rightarrow 1 / 4$.

As a consequence, $\tilde{g}$ as given by (26) tends to $\tilde{g}_{\ell}$ in $L^{2}(\mathbb{R})$ as $\lambda \rightarrow \lambda_{\ell}$, where

$$
\tilde{g}_{\ell}(x)=\sum_{n \in \mathbb{Z}} g(n / \ell) \Phi_{\ell, \text { int }}(x-n / \ell)
$$

Since inequality (34) continues to hold as $\lambda \rightarrow \lambda_{\ell}, \tilde{g} \rightarrow \tilde{g}_{\ell}$, it follows that

$$
\left|g(x)-\tilde{g}_{\ell}(x)\right|^{2} \leq C \ell^{-s} \text { a.e. }
$$

for any $\ell \in \mathbb{N}$ held constant. Because $g, \tilde{g}$ are continuous functions, inequality (35) holds everywhere in $\mathbb{R}$. By inspection of the Fourier transform of $\tilde{g}_{\ell}$ it can be seen that always $\tilde{g}_{\ell} \in \mathcal{P}_{\varphi}$ (whereas $\Phi_{\ell \text {,int }} \notin \mathcal{P}_{\varphi}, \ell=2,3, \ldots !$ ). As a result, the functions $\tilde{g}_{\ell}$ form an approximation to $g$ in $\mathcal{P}_{\varphi}$ with sth-order decay of squared error $\left|g(x)-\tilde{g}_{\ell}(x)\right|^{2}$ as $\ell \rightarrow \infty$, uniformly in $x \in \mathbb{R}$.

Since $\Phi_{\ell, \text { int }}$ is the interpolating function (11) for the dilated prefilter function $\varphi_{\ell}(x)=\ell^{1 / 2} \beta^{m-1}(\ell x)$ and $\lambda=1 / \ell$, it can be computed by the theorem of residues as indicated in [1]. 
There is no reason to refrain from using non-centered B-spline functions $N_{m}(x)$ [23] of any order $m=2,3,4, \ldots$ defined by

$$
\begin{aligned}
N_{m}(x) & =(\underbrace{\chi_{[0,1]} * \ldots * \chi_{[0,1]}}_{m \text { times }})(x)=\beta^{m-1}\left(x-\frac{m}{2}\right) \\
& \stackrel{\text { Fourier }}{\longleftrightarrow} \hat{N}_{m}(\xi)=\frac{1}{\sqrt{2 \pi}}\left(e^{-i \xi / 2} \frac{\sin (\xi / 2)}{\xi / 2}\right)^{m} .
\end{aligned}
$$

Indeed, the prefilter function $\varphi(x)=N_{m}(x)$ will result in the same localization space $\mathcal{P}_{\varphi}$ as when using $\varphi(x)=\beta^{m-1}(x)$. The interpolating functions $\Phi_{\text {int }}$ will also coincide. Therefore, when $\varphi(x)=N_{m}(x)$ we always have a sampling theorem in the space $\mathcal{R}_{\lambda}(\varphi)=\boldsymbol{P}_{\varphi} V_{\lambda}(\varphi)$ (at least when $0<\lambda \neq \frac{1}{2}, \frac{1}{3}, \frac{1}{4}, \ldots$ ). On the other hand, there is no sampling theorem in the shift-invariant subspace $V_{\lambda}(\varphi) \subseteq L^{2}(\mathbb{R})$ in case $\lambda=1$ and $m=3,5,7, \ldots$ as shown by the following counterexample (a simple generalization of the one given by Walter [30]): The interpolating function (see Remark 2) $\varphi_{\text {int }} \in V_{1}\left(N_{m}\right), m \geq 3$ odd, defined by

$$
\hat{\varphi}_{\text {int }}(\xi)=\frac{\hat{N}_{m}(\xi)}{\sqrt{2 \pi} \sum_{n \in \mathbb{Z}} \hat{N}_{m}(\xi+2 \pi n)}
$$

has a pole in $\xi=\pi$ because in the numerator we obtain $\hat{N}_{m}(\pi) \neq 0$ whereas the denominator becomes

$$
\sqrt{2 \pi} \sum_{n=-\infty}^{\infty} \hat{N}_{m}(\pi+2 \pi n)=\sum_{n=-\infty}^{\infty}\left[i\left(n+\frac{1}{2}\right) \pi\right]^{-m}=0 .
$$

As a conclusion, in Figure 2 the Fourier transforms of related interpolating functions $\Phi_{\text {int }}$ or $\Phi_{\ell \text {,int }}$ are depicted: for ideal low-pass $(\beta=4)$, Gaussian $(\beta=2)$ and B-spline $(m=3)$ prefilter function $\varphi$ and $\lambda=0.25$ or $\ell=4$.

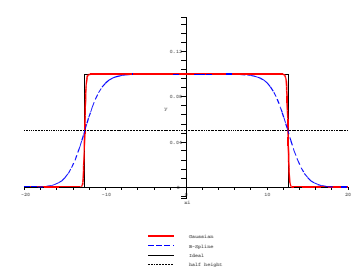

Fig. 2. Interpolating functions in frequency domain. 


\section{ACKNOWLEDGEMENT}

The present work originates in a talk given in the Time-Frequency Seminar of EUCETIFA during a stay at the University of Vienna, May 15-17, 2006. We are grateful to K. Gröchenig and H. G. Feichtinger for hospitality and advice.

\section{References}

[1] A. Aldroubi, M. Unser, and M. Eden, Cardinal spline filters: Stability and convergence to the ideal sinc interpolator, Signal Process., 28, 127-138, 1992.

[2] A. Aldroubi and M. Unser, Sampling procedures in function spaces and asymptotic equivalence with Shannon's sampling theory, Numer. Funct. Anal. and Optimiz., 15, 1-21, 1994.

[3] T. Blu and M. Unser, Approximation error for quasi-interpolators and (multi-)wavelet expansions, Appl. Comput. Harmon. Anal., 6, 219-251, 1999.

[4] C. de Boor, R.A. DeVore, and A. Ron, Approximation from shift-invariant subspaces of $L_{2}\left(\mathbb{R}^{d}\right)$, Trans. Amer. Math. Soc., 341, 787-806, 1994.

[5] P.L. Butzer, W. Splettstösser, and R.L. Stens, The sampling theorem and linear prediction in signal analysis, Jber. d. Dt. Math.-Verein., 90, 1-70, 1988.

[6] P.L. Butzer and R.L. Stens, Sampling theory for not necessarily bandlimited functions: A historical overview, SIAM Review, 34, 40-53, 1992.

[7] O. Christensen, An Introduction to Frames and Riesz Bases, Birkhäuser, Boston, 2003.

[8] I. Daubechies, The wavelet transform, time-frequency localization and signal analysis, IEEE Trans. Inform. Theory, 36, 961-1005, 1990.

[9] Y.C. Eldar, Sampling with arbitrary sampling and reconstruction spaces and oblique dual frame vectors, J. Fourier Anal. Appl., 9, 77-96, 2003.

[10] Y.C. Eldar and T. Werther, General framework for consistent sampling in Hilbert spaces, Int. J. of Wavelets, Multiresolution and Inform. Process., 3, 497-509, 2005.

[11] Y.C. Eldar and T.G. Dvorkind, A minimum squared-error framework for generalized sampling, IEEE Trans. Signal Process., 54, 2155-2167, 2006. 
[12] H.G. Feichtinger and T. Strohmer (ed.), Advances in Gabor Analysis, Birkhäuser, Boston, 2003.

[13] W. Feller, An Introduction to Probability Theory and Its Applications, vol. I, 3rd ed., John Wiley \& Sons, New York, 1967 (revised printing 1970).

[14] D. Gabor, Theory of communication, J. Inst. Elect. Eng. (London), 93 (III), 429-457, 1946.

[15] K. Gröchenig, Foundations of Time-Frequency Analysis, Birkhäuser, Boston, 2001.

[16] E. Hammerich, A sampling theorem for time-frequency localized signals, Sampl. Theory Signal Image Process., 3, 45-81, 2004.

[17] E. Hammerich, Sampling in shift-invariant spaces with Gaussian generator, Sampl. Theory Signal Image Process., 6, 71-86, 2007.

[18] A.J. Jerri, The Shannon sampling theorem - Its various extensions and applications: A tutorial review, Proc. IEEE, 65, 1565-1596, 1977.

[19] A.J. Jerri, Error analysis in application of generalizations of the sampling theorem, in: R.J. Marks II (ed.), Advanced topics in Shannon sampling and interpolation theory, Springer, New York, 219-298, 1993.

[20] H.P. Kramer, A generalized sampling theorem, J. Math. and Phys., 38, 68-72, 1959.

[21] V.G. Maz’ja, Sobolev Spaces, Springer, Berlin, 1985.

[22] M.Z. Nashed and G.G. Walter, General sampling theorems for functions in reproducing kernel Hilbert spaces, Math. Control, Signals, Syst., 4, 363390, 1991.

[23] I.J. Schoenberg, Contribution to the problem of approximation of equidistant data by analytic functions, Quart. Appl. Math., 4, 45-99, 112-141, 1946 .

[24] C.E. Shannon, Communication in the presence of noise, Proc. IRE, 37, 10-21, 1949 .

[25] W. Sun, Sampling theorems for multivariate shift invariant subspaces, Sampl. Theory Signal Image Process., 4, 73-98, 2005.

[26] M. Unser and A. Aldroubi, A general sampling theory for nonideal acquisition devices, IEEE Trans. Signal Process., 42, 2915-2925, 1994. 
[27] M. Unser, Splines: A perfect fit for signal and image processing, IEEE Signal Process. Mag., 16, 22-38, 1999.

[28] M. Unser, Sampling - 50 years after Shannon, Proc. IEEE, 88, 569-587, 2000 .

[29] P.P. Vaidyanathan, Generalizations of the sampling theorem: Seven decades after Nyquist, IEEE Trans. Circuit Syst. I, 48, 1094-1109, 2001.

[30] G.G. Walter, A sampling theorem for wavelet subspaces, IEEE Trans. Inform. Theory, 38, 881-884, 1992.

[31] R.M. Young, An Introduction to Nonharmonic Fourier Series (Revised First Edition), Academic Press, London, 2001. 